\title{
Molecular Dynamics Study of Liquid Rubidium and the Lennard-Jones Fluid
}

\author{
Raymond D. Mountain and Steven W. Haan* \\ Center for Thermodynamics and Molecular Science, National Bureau of Standards, Washington, DC 20234 \\ July 11,1979
}

\begin{abstract}
Molecular dynamics calculations were made for three thermodynamic states of a model of liquid rubidium and for two states of the Lennard-Jones fluid in order to investigate the influence of density, temperature and interatomic potential on the spectra of density fluctuations in these fluids. Here the results for the intermediate scattering function, the radial distribution function, the velocity autocorrelation function and the transverse momentum autocorrelation function are presented in tabular form. The procedures employed in this study are discussed and the major features of these functions are described.
\end{abstract}

Key words: Intermediate scattering function; Lennard-Jones liquid; liquid rubidium; molecular dynamics; radial distribution function; transverse current correlation function; velocity autocorrelation function.

\section{Introduction}

One of the most important ways of studying collective effects in liquids is the observation of density fluctuations $[1] .{ }^{1}$ These can be observed experimentally using inelastic coherent neutron scattering. Molecular dynamics computer simulations also provide a way of studying density fluctuations, since the simulations allow one to calculate the fluctuations in model systems. Studies using these techniques have revealed a striking difference between the dynamical properties of liquid rubidium and the rare gas fluids: liquid rubidium has been found to support sound modes with wavelengths only $1^{1 / 4}$ times the interparticle separation, both experimentally [2] and in simulations [3], while the rare gas liquids [4] and the Lennard-Jones liquid [5] cannot support sound waves with wavelengths less than four times the interparticle separation.

We have used molecular dynamics to study the properties of these two systems - simulated rubidium and the LennardJones fluid. The liquid states which we studied were chosen so that we could determine the relative importance of the density, the temperature, and the interatomic potential as the variables which could be responsible for the remarkable difference between these fluids. Our conclusion was that the interatomic potential is the main source of this difference. The analysis which led to this conclusion is presented in ref. [6].

In this article we present our results for various quantities describing the microscopic properties of these liquids, as well as tabulated values of the functions considered in ref. [6]. The quantities which we have evaluated are: $g_{2}(r)$, the

* NBS-NRC Postdoctoral Research Associate

${ }^{1}$ Figures in brackets refer to literature references at the end of this paper. pair correlation function; $F(Q, t)$, the intermediate scattering function; $\psi(t)$, the single particle velocity autocorrelation function; and $C_{T}(Q, t)$, the time autocorrelation of the transverse momentum current. An analysis of the relative motion of pairs of atoms, which is based on these molecular dynamics coordinates, is presented in ref. [7].

We studied a relatively small system of 250 atoms. This means that some details - for example the line shape of the sound modes - were not properly accessible in this study. On the other hand, using a smaller system made it feasible to perform more calculations, so that we could systematically vary the density and temperature.

The states which we examined can be specified by giving the reduced density and temperature. The reduced density is defined by

$$
n^{*}=N \sigma^{3} / V
$$

where $N$ atoms are in volume $V$ and the length $\sigma$ corresponds to the smallest zero of the pair potential. The reduced temperature is defined by scaling the temperature with the potential well depth $\epsilon$,

$$
T^{*}=k_{B} T / \epsilon,
$$

where $k_{B}$ is Boltzmann's constant. The three states of rubidium which we simulated are listed in table 1 . These were selected to complement Rahman's investigation of the state with $n^{*}=.905$ and $T^{*}=.793$. [3] The two LennardJones states which we examined, listed in table 2, were selected to complement the rubidium simulation and the simulation of Levesque et al. [5], who studied the LennardJones liquid with $n^{*}=.8442$ and $T^{*}=.722$.

We used the effective pair potential developed by Price et 
TABLE 1. Rubidium parameters

\begin{tabular}{crlccc}
\hline \hline State Label & $n^{*}$ & \multicolumn{1}{c}{$T^{*}$} & $\epsilon / k_{B}(K)$ & $\sigma(\AA)$ & $\Delta t(s)$ \\
\hline RB1 & 0.951 & 0.878 & 393 & 4.480 & $2.207 \times 10^{-14}$ \\
RB2 & .951 & 1.11 & 393 & 4.480 & $2.207 \times 10^{-14}$ \\
RB3 & .855 & 1.49 & 407 & 4.482 & $2.288 \times 10^{-14}$ \\
\hline
\end{tabular}

TABLE 2. Lennard-Jones parameters. $\Delta$ t assumes Argon mass and parameters $\left(\sigma=3.4 \AA\right.$ and $\left.\epsilon=120 k_{B}\right)$

\begin{tabular}{clrc}
\hline \hline State Label & $T^{*}$ & $n^{*}$ & $\Delta t(s)$ \\
\hline LJ1 & 0.982 & 0.950 & $1.453 \times 10^{-14}$ \\
LJ2 & 1.31 & .950 & $1.453 \times 10^{-14}$ \\
\hline
\end{tabular}

al. [8] to represent the interaction between the rubidium ions. Rahman [3] showed that this potential provides a faithful representation of density fluctuations in the liquid rubidium state which was studied by neutron scattering in ref. [2]. It is therefore likely that our results for the other states are also appropriate to the physical system. A brief description of how the potentials were constructed is in ref. [9]. In figure 1 we show the effective pair potential for rubidium at a reduced density $n^{*}=0.855$. The potential used for rubidium at $n^{*}=.951$ would be almost indistinguishably different if it were plotted in figure 1 . The other potential which we used, the Lennard-Jones 12-6, provides results which are generally taken to be typical of the rare gas liquids but are not those of any real liquid. The molecular dynamics calculations were performed on a collection of 250 particles with cubical periodic boundary conditions. The particles were assumed to interact through a pair-wise

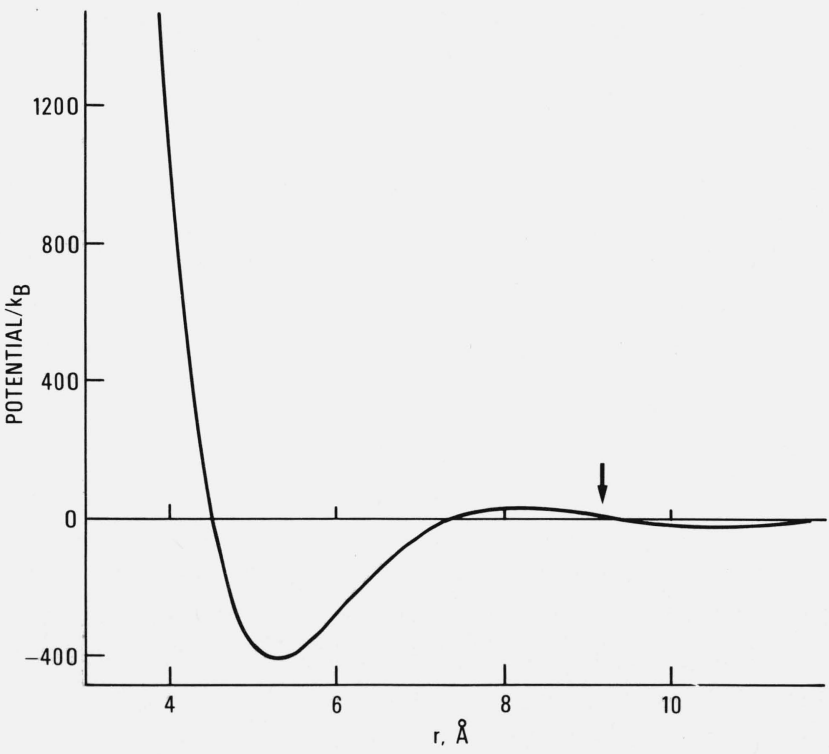

FiguRE 1. Effective pair potential for liquid rubidium state RB3.

On the scale, the potentials for the other density would differ from this one only in the region $4.5 \AA-7 \AA$ where it would lie about one line width above this curve. The arrow indicates where the potential was truncated. additive potential truncated at about $2 \sigma$. The arrow in figure 1 indicates where the rubidium potential for density $n^{*}=$ .855 was truncated. Newton's equations of motion for the 250 particles were solved using an iterative procedure developed by Tsai. [10] This scheme conserves the energy and the momentum of the system. The time steps used in the integration procedure are listed in tables 1 and 2 . The time scale in table 2 is determined by assuming that the atoms have the atomic mass of Argon.

The input needed for a calculation includes the potential, the density, initial values for positions and velocities of the particles, and the energy. The energies were obtained from Monte Carlo calculations of the internal energy for the densities and temperatures of interest [11]. Initial values for the positions and velocities were obtained by taking values from an earlier liquid state simulation, rescaling the velocities, and integrating the equations of motion with the new potential until the kinetic energy stabilized around the desired value. We found this to be a good indication that equilibrium conditions have been achieved provided that spatially disordered initial positions were used. The positions and velocities resulting from this process were taken as initial conditions for our simulation. Each simulation consisted of 2000 time steps of length $\Delta t$. The coordinates and velocities were stored on magnetic tape for subsequent analysis.

The next section contains a description of how the quantities were constructed, tables of the results and a discussion of the major features of each function.

\section{Results}

\subsection{Radial distribution function}

We evaluated the equilibrium radial distribution function [12] for each fluid by determining the distance between each pair of particles (minimized by considering replicas of the particles as generated by the periodic boundary conditions). These distances were then sorted into bins of length $0.01 \sigma$. The bin counts were accumulated over 600 sequential time steps and then appropriately normalized. The results are presented in table 3 . The number of counts in one bin varied from $10^{4}$ to $10^{5}$ so that the statistical errors are on the order of 1 percent. The largest $r$ for which we could sensibly evaluate the pair correlation function $g_{2}(r)$, is one-half the period of the spatial boundary condition; this maximum is approximately $3.2 \sigma$ for these densities.

The radial distribution functions for these fluids do not differ strikingly from each other. The only readily apparent difference is that $g_{2}(r)$ for a Lennard-Jones fluid shows a more rapid initial increase from zero and a sharper first maximum than the $g_{2}(r)$ for any of the rubidium states. This is a natural result of the harder core of the Lennard-Jones potential. 
TAELE 3, RADIAL DISTRIBUTION FUNCTIONS, R IS IN UNITS OF SIGMA,

STATE

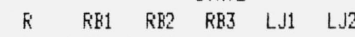

$\begin{array}{lllllll}0.70 & 0.000 & 0.000 & 0.001 & 0.000 & 0.000\end{array}$

$\begin{array}{lllllll}0.72 & 0.000 & 0.000 & 0.001 & 0.000 & 0.000\end{array}$

$\begin{array}{lllllll}0.74 & 0.000 & 0.001 & 0.003 & 0.000 & 0.000\end{array}$

$\begin{array}{lllllllll}0.76 & 0.000 & 0.004 & 0.009 & 0.000 & 0.000\end{array}$

$\begin{array}{llllll}0.78 & 0.000 & 0.010 & 0.028 & 0.000 & 0.000\end{array}$

$\begin{array}{lllllll}0.80 & 0.005 & 0.020 & 0.051 & 0.000 & 0.000\end{array}$

$\begin{array}{llllllllllllll}0.82 & 0.015 & 0.048 & 0.104 & 0.000 & 0.000\end{array}$

$\begin{array}{llllllllllllll}0.84 & 0.038 & 0.112 & 0.178 & 0.000 & 0.001\end{array}$

$\begin{array}{llllllllllllllllll}0.86 & 0.092 & 0.209 & 0.289 & 0.000 & 0.007\end{array}$

$\begin{array}{llllllllllll}0.88 & 0.185 & 0.332 & 0.442 & 0.001 & 0.023\end{array}$

$\begin{array}{lllllllllllll}0.90 & 0.342 & 0.533 & 0.609 & 0.013 & 0.064\end{array}$

$\begin{array}{lllllll}0.92 & 0.565 & 0.798 & 0.832 & 0.089 & 0.240\end{array}$

$\begin{array}{lllllll}0.94 & 0.841 & 1.090 & 1.062 & 0.346 & 0.671\end{array}$

$\begin{array}{lllllll}0.96 & 1.194 & 1.451 & 1.309 & 0.875 & 1.301\end{array}$

$\begin{array}{lllllll}0.98 & 1.536 & 1.726 & 1.501 & 1.618 & 1.951\end{array}$

$\begin{array}{lllllll}1.00 & 1.911 & 1.911 & 1.677 & 2.369 & 2.488\end{array}$

$\begin{array}{llllll}1,02 & 2.193 & 2,116 & 1,819 & 2.832 & 2,830\end{array}$

$\begin{array}{lllllll}1.04 & 2.390 & 2.228 & 1.937 & 3.158 & 2.961\end{array}$

$\begin{array}{lllllll}1.06 & 2.494 & 2.315 & 1.945 & 3.192 & 2.848\end{array}$

$\begin{array}{lllllll}1.08 & 2.536 & 2.286 & 1.961 & 3.042 & 2.671\end{array}$

$\begin{array}{lllllll}1.10 & 2.508 & 2.210 & 1.935 & 2.764 & 2.468\end{array}$

$\begin{array}{lllllll}1.12 & 2.398 & 2.132 & 1.915 & 2.503 & 2.219\end{array}$

$\begin{array}{lllllll}1.14 & 2.234 & 1.999 & 1.797 & 2.144 & 1.973\end{array}$

$\begin{array}{llllllll}1.16 & 2.056 & 1.815 & 1.715 & 1.917 & 1.753\end{array}$

$\begin{array}{llllllll}1.18 & 1.870 & 1.689 & 1.645 & 1.644 & 1.584\end{array}$

$\begin{array}{llllllll}1.20 & 1.677 & 1.547 & 1.552 & 1.454 & 1.411\end{array}$

$\begin{array}{lllllll}1.22 & 1.503 & 1.418 & 1.478 & 1.289 & 1.225\end{array}$

$\begin{array}{lllllll}1.24 & 1.311 & 1,283 & 1.339 & 1.137 & 1.132\end{array}$

$\begin{array}{lllllll}1.26 & 1.182 & 1.148 & 1.286 & 0.973 & 1.019\end{array}$

$\begin{array}{lllllll}1.28 & 1,058 & 1.054 & 1.190 & 0.881 & 0.911\end{array}$

$\begin{array}{llllllllllllllll}1.30 & 0.939 & 0.968 & 1.087 & 0.790 & 0.850\end{array}$

$\begin{array}{lllllll}1.32 & 0.828 & 0.905 & 1.042 & 0.744 & 0.779\end{array}$

$\begin{array}{llllllllll}1.34 & 0.768 & 0.838 & 0.974 & 0.686 & 0.715\end{array}$

$\begin{array}{lllllll}1.36 & 0.720 & 0.768 & 0.933 & 0.645 & 0.677\end{array}$

$\begin{array}{llllllll}1.38 & 0.678 & 0.709 & 0.891 & 0.596 & 0.651\end{array}$

$\begin{array}{lllllll}1.40 & 0.625 & 0.675 & 0.836 & 0.566 & 0.625\end{array}$

$\begin{array}{lllllll}1.42 & 0.583 & 0.644 & 0.810 & 0.553 & 0.586\end{array}$

$\begin{array}{lllllllll}1.44 & 0.550 & 0.637 & 0.779 & 0.525 & 0.573\end{array}$

$\begin{array}{lllllllll}1.46 & 0.539 & 0.624 & 0.740 & 0.532 & 0.573\end{array}$

$\begin{array}{llllllllll}1.48 & 0.519 & 0.603 & 0.728 & 0.522 & 0.585\end{array}$

$\begin{array}{lllllllllllll}1.50 & 0.529 & 0.600 & 0.718 & 0.533 & 0.582\end{array}$

$\begin{array}{lllllll}1.52 & 0.526 & 0.592 & 0.706 & 0.544 & 0.573\end{array}$
STATE

$\begin{array}{llllll}R & R B 1 & R B 2 & R B 3 & L J 1 & L J 2\end{array}$ $\begin{array}{lllllllll}1.54 & 0.534 & 0.599 & 0.694 & 0.540 & 0.588\end{array}$ $\begin{array}{llllllllllll}1.56 & 0.547 & 0.610 & 0.707 & 0.560 & 0.590\end{array}$ $\begin{array}{lllllllllll}1.58 & 0.550 & 0.620 & 0.714 & 0.574 & 0.611\end{array}$ $\begin{array}{lllllllll}1.60 & 0.583 & 0.651 & 0.718 & 0.605 & 0.643\end{array}$ $\begin{array}{lllllllllll}1.62 & 0.615 & 0.672 & 0.716 & 0.641 & 0.663\end{array}$ $\begin{array}{llllllll}1.64 & 0.652 & 0.693 & 0.735 & 0.673 & 0.698\end{array}$ $\begin{array}{lllllll}1.66 & 0.695 & 0.723 & 0.756 & 0.719 & 0.744\end{array}$ $\begin{array}{llllllllllll}1.68 & 0.729 & 0.750 & 0.778 & 0.768 & 0.796\end{array}$ $\begin{array}{lllllllll}1.70 & 0.762 & 0.802 & 0.795 & 0.814 & 0.844\end{array}$ $\begin{array}{lllllllll}1.72 & 0.810 & 0.823 & 0.827 & 0.865 & 0.884\end{array}$ $\begin{array}{lllllllllllllllll}1.74 & 0.865 & 0.879 & 0.879 & 0.917 & 0.920\end{array}$ $\begin{array}{lllllllll}1.76 & 0.937 & 0.920 & 0.898 & 0.962 & 0.978\end{array}$ $\begin{array}{llllllllllllll}1.78 & 0.983 & 0.972 & 0.907 & 1.030 & 1.025\end{array}$ $\begin{array}{llllllll}1.80 & 1.032 & 1.014 & 0.936 & 1.078 & 1.047\end{array}$ $\begin{array}{llllll}1.82 & 1.075 & 1.064 & 0.953 & 1.119 & 1.089\end{array}$ $\begin{array}{llllll}1.84 & 1.101 & 1.079 & 0,985 & 1.156 & 1.125\end{array}$ $\begin{array}{llllll}1.86 & 1.167 & 1.127 & 1.015 & 1.170 & 1.174\end{array}$ $\begin{array}{llllll}1.88 & 1.190 & 1.154 & 1.037 & 1.222 & 1.196\end{array}$ $\begin{array}{lllllll}1.90 & 1.221 & 1.191 & 1.073 & 1.237 & 1.222\end{array}$ $\begin{array}{lllllll}1.92 & 1.249 & 1.212 & 1.098 & 1.257 & 1.238\end{array}$ $\begin{array}{lllllll}1.94 & 1.265 & 1.237 & 1.107 & 1.277 & 1.244\end{array}$ $\begin{array}{lllllll}1.96 & 1.294 & 1.250 & 1.112 & 1.275 & 1.278\end{array}$

$\begin{array}{llllll}1.98 & 1.315 & 1.265 & 1.140 & 1.280 & 1.281\end{array}$

$\begin{array}{lllllll}2.00 & 1.317 & 1.269 & 1.169 & 1.292 & 1.274\end{array}$

$2,02 \quad 1.303 \quad 1.2551,164 \quad 1,3001.267$

$\begin{array}{llllllll}2.04 & 1.304 & 1.250 & 1.170 & 1.290 & 1.243\end{array}$

$\begin{array}{lllllll}2.06 & 1.288 & 1.238 & 1.171 & 1.291 & 1.241\end{array}$

$2.08 \quad 1.261 \quad 1.209 \quad 1.165 \quad 1.253 \quad 1.237$

$2.10 \quad 1.240 \quad 1.191 \quad 1.175 \quad 1.251 \quad 1.203$

$2.12 \quad 1.222 \quad 1.164 \quad 1.154 \quad 1.224 \quad 1.191$

$\begin{array}{lllllll}2.14 & 1.202 & 1.140 & 1.130 & 1.190 & 1.137\end{array}$

$\begin{array}{lllllll}2.16 & 1.155 & 1.135 & 1.124 & 1.150 & 1.097\end{array}$

$\begin{array}{lllllllll}2.18 & 1.120 & 1.088 & 1.114 & 1.095 & 1.066\end{array}$

$2.20 \quad 1.088 \quad 1.064 \quad 1.094 \quad 1.0551 .032$

$\begin{array}{lllllll}2.22 & 1.049 & 1.036 & 1.079 & 1.021 & 0.992\end{array}$

$2.24 \quad 1.014 \quad 1.011 \quad 1.0590 .983 \quad 0.971$

$\begin{array}{lllllllll}2.26 & 0.987 & 0.981 & 1.046 & 0.939 & 0.940\end{array}$

$\begin{array}{lllllllll}2.28 & 0.954 & 0.951 & 1.016 & 0.897 & 0.923\end{array}$

$\begin{array}{lllllll}2.30 & 0.915 & 0.942 & 1.007 & 0.885 & 0.892\end{array}$

$\begin{array}{lllllllll}2.32 & 0.900 & 0.913 & 0.990 & 0.856 & 0.891\end{array}$

$\begin{array}{lllllllll}2.34 & 0.873 & 0.893 & 0.978 & 0.830 & 0.861\end{array}$

$\begin{array}{lllllllll}2.36 & 0.841 & 0.885 & 0.957 & 0.813 & 0.845\end{array}$
STATE

$\begin{array}{llllll}R & R B 1 & R B 2 & R B 3 & L J 1 & L J\end{array}$ $\begin{array}{lllllllll}2.38 & 0.827 & 0.877 & 0.958 & 0.788 & 0.832\end{array}$

$\begin{array}{llllllll}2.40 & 0.810 & 0.866 & 0,941 & 0.792 & 0.817\end{array}$

$\begin{array}{llllllll}2.42 & 0.803 & 0.847 & 0.923 & 0.788 & 0.812\end{array}$

$\begin{array}{llllllll}2.44 & 0.796 & 0.848 & 0.924 & 0.783 & 0.826\end{array}$

$\begin{array}{llllllllll}2.46 & 0.804 & 0.843 & 0.914 & 0.796 & 0.836\end{array}$

$\begin{array}{lllllll}2.48 & 0.786 & 0.853 & 0.922 & 0.793 & 0.836\end{array}$

$\begin{array}{llllllllll}2.50 & 0.792 & 0.837 & 0.916 & 0.803 & 0.854\end{array}$

$\begin{array}{llllllll}2.52 & 0.796 & 0.855 & 0.914 & 0.815 & 0.868\end{array}$

$\begin{array}{lllllll}2.54 & 0.825 & 0.877 & 0.915 & 0.837 & 0.884\end{array}$

$\begin{array}{llllllllll}2.56 & 0.842 & 0.891 & 0.909 & 0.870 & 0.885\end{array}$

$\begin{array}{llllllllll}2.58 & 0.865 & 0.896 & 0.924 & 0.887 & 0.908\end{array}$

$\begin{array}{llllllll}2.60 & 0.869 & 0.908 & 0.926 & 0.915 & 0.928\end{array}$

$\begin{array}{llllllll}2.62 & 0.895 & 0.929 & 0.936 & 0.932 & 0.951\end{array}$

$\begin{array}{lllllllll}2.64 & 0.925 & 0.948 & 0.944 & 0.959 & 0.970\end{array}$

$\begin{array}{llllllll}2.66 & 0.954 & 0.963 & 0.947 & 0.998 & 0.988\end{array}$

$\begin{array}{lllllllll}2.68 & 0.985 & 0.974 & 0.953 & 1.017 & 1.002\end{array}$

$2.70 \quad 1,005 \quad 0.994 \quad 0.971 \quad 1.0361 .019$

$\begin{array}{lllllllll}2.72 & 1.031 & 1.003 & 0.968 & 1.053 & 1.036\end{array}$

$\begin{array}{lllllll}2.74 & 1.049 & 1.017 & 0.969 & 1.066 & 1.055\end{array}$

$\begin{array}{llllllll}2.76 & 1.067 & 1.049 & 0.974 & 1.095 & 1.084\end{array}$

$2.78 \quad 1.088 \quad 1.045 \quad 1.004 \quad 1.1051 .078$

$2.80 \quad 1.1101 .066 \quad 1.005 \quad 1.121 \quad 1.098$

$2.82 \quad 1.113 \quad 1.0741 .0101 .1151 .09$ !

$2.84 \quad 1.123 \quad 1.091 \quad 1.025 \quad 1.1321 .104$

$\begin{array}{lllllll}2.86 & 1.134 & 1.088 & 1.027 & 1.135 & 1.114\end{array}$

$\begin{array}{llllll}2.88 & 1.141 & 1.099 & 1.031 & 1.130 & 1.108\end{array}$

$2.90 \quad 1.136 \quad 1.102 \quad 1.033 \quad 1.138 \quad 1.099$

$\begin{array}{lllllll}2.92 & 1.138 & 1.097 & 1.042 & 1.133 & 1.104\end{array}$

$2.94 \quad 1.128 \quad 1.084 \quad 1.043 \quad 1.122 \quad 1.105$

$\begin{array}{lllllll}2.96 & 1.126 & 1.084 & 1.042 & 1.115 & 1.091\end{array}$

$2.98 \quad 1.117 \quad 1.087 \quad 1.0391 .0901 .090$

$\begin{array}{lllllll}3.00 & 1.113 & 1.087 & 1.048 & 1.084 & 1.078\end{array}$

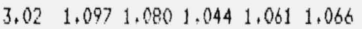

$\begin{array}{llllll}3.04 & 1.087 & 1.068 & 1.044 & 1.065 & 1.059\end{array}$

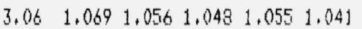

$\begin{array}{lllll}3.08 & 1.048 & 1.0501 .043 \quad 1.028 & 1.029\end{array}$

$3.10 \quad 1.039 \quad 1.044 \quad 1.034 \quad 1.0221 .006$

$\begin{array}{llllll}3.12 & 1.0 .31 & 1.023 & 1.034 & 1.009 & 1.004\end{array}$

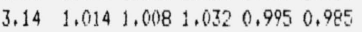

$\begin{array}{llllllll}3.16 & 0.999 & 1.000 & 1.029 & 0.990 & 0.977\end{array}$

$\begin{array}{llllll}3.18 & 0.989 & 0.984 & 1.020 & 0.975 & 0.975\end{array}$

\subsection{Intermediate scattering function}

The intermediate scattering function $F(Q, t)$ is the time autocorrelation function of the spatial Fourier component of the density wavevector $Q$. It is constructed from molecular dynamics data by evaluating

$$
F(Q, t)=\left\langle N_{Q}(\tau+t) N_{-Q}(\tau)\right\rangle
$$

where

$$
N_{Q}(t)=\frac{1}{\sqrt{N}} \sum_{j=1}^{N} \exp \left[i \underline{Q} \cdot \underline{r}_{j}(t)\right]
$$

is the $Q$ th Fourier component of the number density at time $t$ and the angular brackets $\langle\cdots\rangle$ indicate an average over time origins $\tau$. The wavevector $Q$ must be consistent with the periodic boundary conditions. We took $Q$ 's parallel to the $x$, $y$, and $z$ axes of the cubical system. In each direction we considered 10 magnitudes,

$$
Q_{j}=2 \pi j / L, \quad j=1,2, \cdots, 10,
$$

where $L$ is the size of the system. For given $Q$, we first evaluated $N_{Q}(t)$ for each of the 2000 available $t$ values. These coefficients were then used to construct products $N_{Q}(t+\tau) N_{-Q}(\tau)$ for 180 time origins $\tau$, with the origins separated by 10 time steps. The average of these products is our estimate for $F(Q, t)$. The averages for a given $Q_{j}$ involved products over 180 time origins and over the three $Q$ 's parallel to the cube axes, yielding a total of 540 samples. With this size sample, the statistical fluctuations about zero as $t \rightarrow \infty$ are expected to be on the order of 0.05 . The maximum time interval considered was $100 \Delta t$, the approximate time for a sound wave to cross the cubical system. If longer times are considered, spurious correlations are introduced into $F(Q, t)$. 
Our results are presented in tables $4-8$. The first row of each table contains $F(Q, O)$. The subsequent rows list $F(Q, t) / F(Q, O)$ at intervals of $5 \Delta t$. This time resolution is sufficient to represent the main features of the functions. Intermediate values can be reliably estimated by three point interpolation procedures.

Coherent neutron scattering determines $S(Q, \omega)[1]$, the cosine transform of $F(Q, t)$ :

$$
S(Q, \omega)=\int_{0}^{\infty} d t F(Q, t) \cos \omega t
$$

Some features of $S(Q, \omega)$ for these states have been discussed elsewhere [6]. For the three rubidium states, the oscillations in $F(Q, t)$ for $Q<1.15 \AA^{-1}$ lead to maxima in $S(Q, \omega)$ for $\omega$ $\neq 0$. This is evidence for sound modes with wavelengths as small as $1^{1 / 4}$ the interparticle separation. On the other hand, for the Lennard-Jones states, only the results at the smallest $Q$ show evidence for a sound mode. Transforming the $F(Q, t)$ 's was complicated by considerable noise for $t \geq$ $100 \Delta t$.

We have not attempted further line shape studies as the details are obscured by this noise.
TAELE 4, INTEFIMEIITATE SCATTERING FIJNCTIONS FOR RE1. VALIJES FDF: $T$ O ARE NORMALIZEII TO F(Q.0). THE TIME IS IH UNITS OF $\Delta T$.

WAUE VECTOF O IN INUERSE ANGSTROM UNITS.

$\begin{array}{lllllllllllll}.219 & .438 & .657 & .876 & 1.095 & 1.314 & 1.533 & 1.752 & 1.971 & 2.190\end{array}$

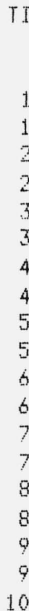

ME

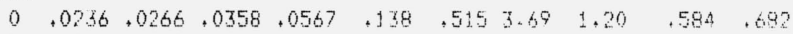

$\begin{array}{llllllllll}5.961 & .869 & .776 & .750 & .820 & .942 & .999 & .946 & .965 & .092\end{array}$

$\begin{array}{llllllllllll}10 & .847 & .538 & .285 & .213 & .416 & .800 & .955 & .822 & .570 & .591\end{array}$

$15.675+160-.106-.175+055+632.911+680+293.369$

$20 \quad .468-.111-.167-.192-.110 \quad .482 \quad .866 \quad .555 \quad .124 \quad .266$

$\begin{array}{lllllllllll}25 & .252-.186 & .041 & .019 & -.079 & .367 & .825 & .45 & .051 & .233\end{array}$

$\begin{array}{llllllllllll}30 \quad .050-.076 & .275 & .196 & .052 & .292 & .788 & .384 & .033 & .213\end{array}$

$35-.116+129+336+212+169+247 \quad .757+330+033+184$

$\begin{array}{lllllllllllll}40 & -.232 & .313 & +212 & .112 & .211 & .225 & .729 & .291 & .026 & .154\end{array}$

$\begin{array}{lllllllllll}45 & -.290 & .392 & .037 & .018 & .185 & .219 & .704 & .261 & .010 & .145\end{array}$

$50-.292 \quad .341-.045-.001,117 \quad .219 \quad .681 \quad .239 \cdots, 009, .149$

$\begin{array}{llllllllllll}55 & -.242 & .202 & .003 & .031 & .043 & .225 & .059 & .218 & -.025 & .167\end{array}$

$60 \quad-.154,047, .110,068-.008+231+638+198-.025, .178$

$\begin{array}{lllllllllll}65 & -.041 & -.054 & .179 & .078 & -.026 & .233 & .618 & .180 & -.019 & .171\end{array}$

$70 \quad .080-.065+170 \quad .064-.013+229 \quad .599+171-.017 \quad .156$

$75 \quad .194 \quad .001 \quad .110 \quad .048 \quad .012 \quad .217 \quad .582 \quad+169-.016 \quad+140$

$\begin{array}{llllllllllll}80 & .289 & .099 & .058 & .029 & .034 & .199 & .566 & .174 & \cdots .003 & .127\end{array}$

$\begin{array}{lllllllllllll}85 & .354 & .178 & .047 & .000 & .040 & .173 & .552 & .177 & .023 & .123\end{array}$

$90+385+203 \quad .061-.029+029+145+538+177+058+115$

$95 \quad .379 \quad .168 \quad .072 \quad-.040 \quad .011 \quad .113 \quad .527 \quad .170 \quad .085 \quad .108$

$\begin{array}{llllllllllll}100 \quad .341 & .097 & .062 & -.022 & -.004 & .084 & .509 & .161 & .098 & .093\end{array}$

TAELE 5, INTERMEIIATE SCATTERING FUNCTIONS FOF RB2, VALUES FOF: $T$ T O ARE NOFMALIZEII TO $F(0,0)$. THE TIME IS IN UNITS OF $\triangle T$.

WAVE VECTOR Q IN INUERSE ANGSTROM UNITS.

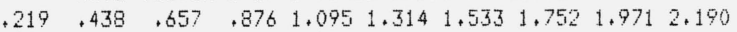

TIME:

$\begin{array}{lllllllllll}0 & .0327 & .0341 & .0444,0688 & .168 & .612 & 2.14 & 1.32 & .706 & .725\end{array}$

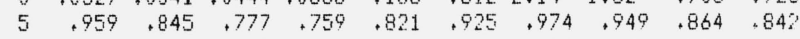

$\begin{array}{llllllllllll}10 & .836 & .462 & .281 & .272 & .423 & .733 & .905 & .823 & .570 & .520\end{array}$

$\begin{array}{lllllllllllll}15 & .661 & .034 & -.0118 & -.063 & .072 & .515 & .825 & .681 & .309 & .288\end{array}$

$20 \quad .444-.265-.198-.089-.087 \quad .313 \quad .741 \quad .552 \quad .157 \quad .192$

$25 \quad .228-.345-.018 \quad .051-.078 \quad .164 \quad .666 \quad .447 \quad .100 \quad . .55$

$\begin{array}{llllllllllll}30 & .023 & -.234 & .199 & .148 & .012 & .067 & .597 & .364 & .083 & .150\end{array}$

$\begin{array}{lllllllllll}35 & -.139 & -.024 & .280 & .109 & .087 & .021 & .535 & .303 & .080 & .161\end{array}$

$\begin{array}{llllllllllllll}40 & -.252 & .160 & .189 & -.002 & .121 & .006 & .481 & .253 & .084 & .149\end{array}$

$\begin{array}{llllllllllll}45 & -.303 & .251 & .018 & -.064 & .118 & .016 & .433 & .220 & .081 & +128\end{array}$

$\begin{array}{llllllllllllll}50 & -.301 & .226 & -. .104 & -.042 & .082 & .029 & .391 & .187 & .083 & .111\end{array}$

$\begin{array}{llllllllllll}55 & -.246 & .124 & -.131 & .016 & .036 & .047 & .355 & .162 & .088 & .089\end{array}$

$\begin{array}{llllllllllll}60 & -.158 & .005 & -.072 & .044 & -.002 & .049 & .324 & .138 & .083 & .084\end{array}$

$65 \quad-.044-.078 \quad .011 \quad .025-.015 \quad .042 \quad .299 \quad .123 \quad .071 \quad .081$

$\begin{array}{lllllllllll}70 & .072 & -.101 & .053 & -.023 & .007 & .021 & .281 & .113 & .059 & .086\end{array}$

$\begin{array}{llllllllll}75 & .181-.070 \quad .046-.064 & .045-.005 & .268 & .041 & .041 & .083\end{array}$

$\begin{array}{llllllllllll}80 & .268 & -.016 & .015 & -.062 & .063 & -.021 & .258 & .117 & .027 & .052\end{array}$

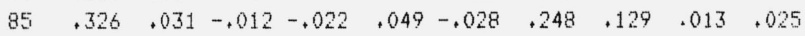

$\begin{array}{lllllllllllll}90 & .349 & .049 & -.016 & .015 & .007 & -.024 & .237 & .137 & .012 & .011\end{array}$

$\begin{array}{lllllllllll}95 & .340 & .033 & -.009 & .017 & -.031 & -.007 & .224 & .142 & .020 & .011\end{array}$

$100 \quad .297-.007-.006-.003-.035 \quad .010 \quad .207 \quad .144 \quad .023 \quad .016$
TABLE 6. INTERMEIIATE SCATTERING FUINCTIONS TOR REB. WALIIFS FOF: $T ? O$ AFE NOFIMALIZEI TO F(O,O), THE IIME IS IN UNTIS OF $\Delta T$.

WAVE UECTOF Q IN INUEFS ANGSTFOM UNITS.

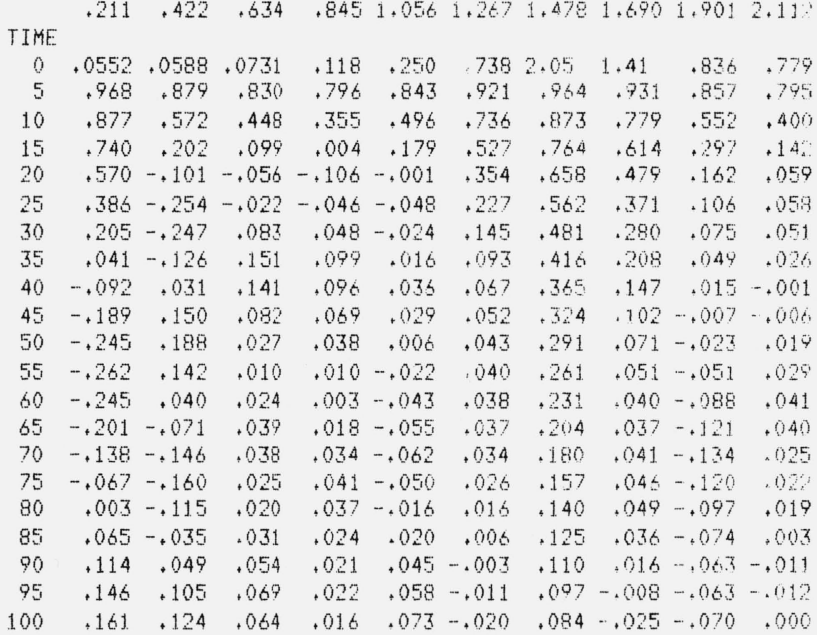

TABLE 7. INTERMEIIATE SCATTEFING FUNCTIONS FOF L.J1. VAL.IJES FQR $T$ $P$ AFE NOFIMALIZEI TO $F(0,0)$. THE TIME IS IN UNITS OF $\Delta \mathrm{T}$.

WAVE VECTOR IN REYUIEI UNITS $(R \sigma)$.

.9801 .9612 .9413 .9224 .9025 .8836 .8637 .8448 .8249 .805

TIME

$\begin{array}{lllllllllll}0 & .0236 & .0231 & .0328 & .0590 & .136 & .463 & 3.58 & 1.52 & .708 & .572\end{array}$

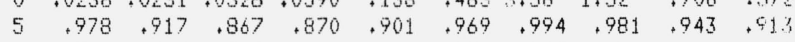

$\begin{array}{llllllllllll}10 & .914 & .707 & .569 & .584 & .681 & .885 & .978 & .926 & .809 & .723\end{array}$

$\begin{array}{lllllllllll}15 & .816 & .460 & .299 & .341 & .458 & .780 & .955 & .857 & .655 & .531\end{array}$

$\begin{array}{llllllllllll}20 & .696 & .260 & .180 & .236 & .307 & .678 & .930 & .786 & .525 & .399\end{array}$

$\begin{array}{llllllllllllll}25 & .565 & .153 & .195 & .229 & .236 & .590 & .904 & .721 & .432 & .324\end{array}$

$\begin{array}{llllllllllll}30+435 & +137+249 & +253 & +220 & +521 & +879 & +664 & .371 & +277\end{array}$

$\begin{array}{lllllllllllllll}35 & .318 & .175 & .276 & .251 & .225 & .466 & .856 & .613 & .333 & .241\end{array}$

$\begin{array}{llllllllllllll}40 & +219 & .221 & .259 & +220 & +232 & +423 & +834 & +567 & .307 & +211\end{array}$

$\begin{array}{lllllllllll}45 & +145 & .244 & .219 & .188 & .236 & .390 & .813 & .526 & .284 & .181\end{array}$

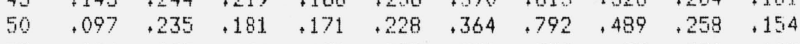

$\begin{array}{lllllllllll}55 & .074+203 & .154 & +176 & +207 & +345 & .773 & +455 & +231 & .127\end{array}$

$\begin{array}{llllllllllllll}60 & .074 & .166 & .137 & .180 & .176 & .328 & .755 & .424 & .209 & .106\end{array}$

$\begin{array}{lllllllllllll}65 & .093 & .139 & .125 & .170 & .140 & .312 & .737 & .397 & .193 & .097\end{array}$

$\begin{array}{lllllllllllll}70 & +125 & .130 & +126 & +142 & .113 & +297 & +720 & +373 & +180 & .099\end{array}$

$\begin{array}{llllllllllllll}75 & +166 & +133 & +134 & .110 & .101 & +281 & .703 & .353 & .167 & .106\end{array}$

$\begin{array}{llllllllllllll}80 & .208 & .143 & .143 & .090 & .101 & .263 & .687 & .336 & +149 & .108\end{array}$

$\begin{array}{llllllllllll}85 & .247 & +151 & .149 & .093 & .108 & .243 & .670 & .324 & +129 & +104\end{array}$

$90 \quad .278+154+145+109+121+221+652+313+105, .098$

$\begin{array}{llllllllllll}95 & .298 & .149 & .129 & .127 & .141 & .197 & .635 & .303 & .082 & .089\end{array}$

$\begin{array}{lllllllllll}100 & .307 & .133 & .107 & .135 & .155 & .174 & .617 & .292 & .059 & .078\end{array}$ 
TAELE 8. INTERMEIIATE SCATTERING FUNCTIONS FOR: L.12, VALUES FOR $T>0$ ARE NORIMALIZEII TO F(Q,0), THE TIME IS IN UNITS OF $\Delta T$.

WAUE VECTOR IN REIUUCE UNITS (QT $\sigma$ ).

.9801 .9612 .9413 .9224 .9025 .8836 .8637 .8448 .8249 .805

TIME

$\begin{array}{lllllllllll}0 & .0293 & .0313 & .0394 & .0726 & .164 & .609 & 2.45 & 1.37 & .768 & .626\end{array}$

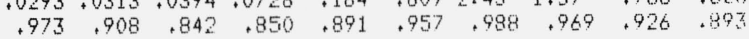

$\begin{array}{llllllllll}.896 & .681 & .500 & .540 & .657 & .856 & .956 & .894 & .761 & .670\end{array}$

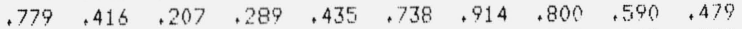

$\begin{array}{llllllllll}.637 & .212 & .088 & .166 & .303 & .631 & .870 & .707 & .462 & .370\end{array}$

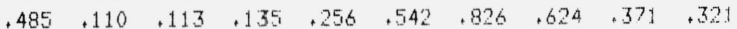

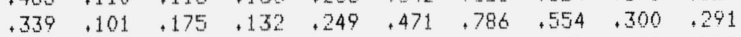

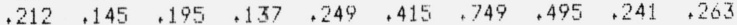

$\begin{array}{llllllllll}.114 & .193 & .175 & .132 & .238 & .370 & .716 & .446 & .190 & .234\end{array}$

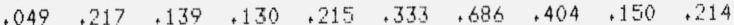

$\begin{array}{llllllllll}.020 & .210 & .115 & .126 & .188 & .303 & .659 & .371 & .123 & .201\end{array}$

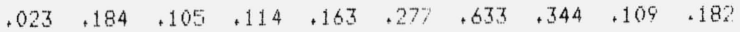

$\begin{array}{llllllllll}.052 & .152 & .101 & .091 & .140 & .248 & .612 & .323 & .110 & .160\end{array}$

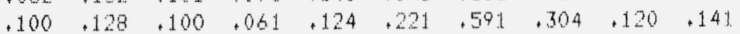

$\begin{array}{llllllllll}.156 & .115 & .104 & .026 & .111 & .191 & .573 & .289 & .125 & .132\end{array}$

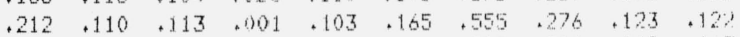

$.260 \quad .108 \quad .119-.011 \quad .100 \quad .141 \quad .538 \quad .266 \quad .119 \quad .113$

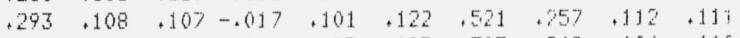

$\begin{array}{llllllllll}.309 & .107 & .073 & \cdots .019 & .105 & .105 & .505 & .249 & .106 & .110\end{array}$

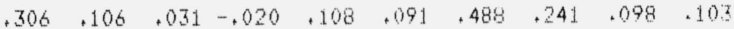

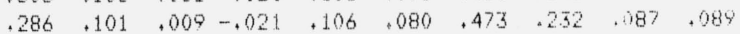

\subsection{Velocity autocorrelation function}

The normalized velocity autocorrelation function is defined as

$$
\psi(t)=\frac{\sum_{j=1}^{N}\left\langle v_{j}(0) \cdot v_{j}(t)\right\rangle}{\sum_{j=1}^{N}\left\langle\left|v_{j}\right|^{2}\right\rangle}
$$

Our results for $\psi(t)$ are presented in table 9. These were obtained by averaging over the 750 velocity components and over 20 time origins separated by 100 time steps. For such an average, the expected fluctuations of $\psi(t)$ about zero as $t$ $\rightarrow \infty$ are on the order of 0.01 .

Representative results are plotted in figure $2 \mathrm{a}$ for rubidium (RB3) and in figure 2b for the Lennard-Jones fluid (LJ1). The functions for rubidium are much more oscillatory than those for the Lennard-Jones fluid. This is in accord with previous observations of other states of these fluids [12, 13] and with theoretical results suggesting a correspondence between oscillating velocity autocorrelation functions and propagating short wavelength sound modes [14].

\subsection{Transverse current correlation function}

One of the characteristics of a liquid is the inability to support a shear stress. However, it is possible to consider transverse momentum currents in liquids since these currents do not involve particle displacements. Rahman showed that,
TABLE 9. Normalized velocity autocorrelation functions. The time is in units of $\Delta t$

\begin{tabular}{c|r|r|r|r|r}
\hline \hline Time & \multicolumn{1}{|c|}{ RB1 } & RB2 & \multicolumn{1}{c|}{ RB3 } & LJ1 & LJ2 \\
\hline 2 & 0.964 & 0.959 & 0.969 & 0.960 & 0.959 \\
4 & 0.858 & 0.847 & 0.874 & 0.849 & 0.837 \\
6 & 0.696 & 0.681 & 0.728 & 0.688 & 0.661 \\
8 & 0.499 & 0.485 & 0.554 & 0.501 & 0.465 \\
10 & 0.291 & 0.282 & 0.372 & 0.314 & 0.277 \\
12 & 0.095 & 0.096 & 0.204 & 0.147 & 0.118 \\
14 & -0.072 & -0.058 & 0.064 & 0.012 & -0.001 \\
16 & -0.198 & -0.167 & -0.040 & -0.087 & -0.079 \\
18 & -0.277 & -0.231 & -0.107 & -0.148 & -0.121 \\
20 & -0.311 & -0.253 & -0.139 & -0.178 & -0.136 \\
22 & -0.305 & -0.239 & -0.143 & -0.182 & -0.132 \\
24 & -0.268 & -0.201 & -0.126 & -0.170 & -0.117 \\
26 & -0.213 & -0.148 & -0.097 & -0.148 & -0.097 \\
28 & -0.151 & -0.093 & -0.063 & -0.122 & -0.078 \\
30 & -0.092 & -0.042 & -0.030 & -0.096 & -0.063 \\
32 & -0.043 & -0.002 & -0.003 & -0.073 & -0.053 \\
34 & -0.010 & 0.024 & 0.017 & -0.055 & -0.048 \\
36 & 0.008 & 0.036 & 0.029 & -0.042 & -0.046 \\
38 & 0.010 & 0.037 & 0.034 & -0.036 & -0.045 \\
40 & 0.009 & 0.028 & 0.032 & -0.034 & -0.042 \\
42 & -0.015 & 0.015 & 0.026 & -0.034 & -0.038 \\
44 & -0.033 & -0.001 & 0.017 & -0.035 & -0.033 \\
46 & -0.048 & -0.015 & 0.007 & -0.037 & -0.028 \\
48 & -0.058 & -0.025 & -0.002 & -0.039 & -0.024 \\
50 & -0.062 & -0.029 & -0.009 & -0.041 & -0.021 \\
52 & -0.058 & -0.028 & -0.014 & -0.042 & -0.019 \\
54 & -0.050 & -0.023 & -0.015 & -0.041 & -0.016 \\
56 & -0.039 & -0.015 & -0.013 & -0.038 & -0.015 \\
58 & -0.026 & -0.006 & -0.007 & -0.034 & -0.014 \\
60 & -0.013 & 0.004 & -0.001 & -0.031 & -0.012 \\
62 & -0.001 & 0.013 & 0.006 & -0.027 & -0.011 \\
64 & 0.008 & 0.019 & 0.011 & -0.024 & -0.010 \\
66 & 0.015 & 0.022 & 0.015 & -0.021 & -0.011 \\
68 & 0.018 & 0.022 & 0.018 & -0.018 & -0.014 \\
70 & 0.018 & 0.021 & 0.018 & -0.016 & -0.018 \\
72 & 0.017 & 0.019 & 0.017 & -0.015 & -0.022 \\
74 & 0.013 & 0.016 & 0.014 & -0.014 & -0.025 \\
76 & 0.010 & 0.013 & 0.010 & -0.013 & -0.025 \\
78 & 0.006 & 0.009 & 0.005 & -0.012 & -0.023 \\
80 & 0.005 & 0.006 & 0.002 & -0.012 & -0.019 \\
\hline & & & & &
\end{tabular}

for sufficiently large wavevectors, an appropriately defined transverse momentum current took on the character of a propagating mode. [15]. The transverse current variable is

$$
J_{T}(\underline{Q}, t)=\frac{1}{\sqrt{N}} \sum_{j=1}^{N} m \underline{v}_{j}^{T}(t) \exp \left[i \underset{Q}{Q} \cdot r_{j}(\underset{\sim}{t})\right]
$$

where $v_{j}^{T}$ is the projection of the velocity of particle $j$ onto the plane perpendicular to $Q$. The transverse current correlation function is then

$$
C_{T}(Q, t)=1 / 2\left\langle J_{T}(\underline{Q}, 0) \cdot \underline{J}_{T}(-\underline{Q}, t)\right\rangle .
$$



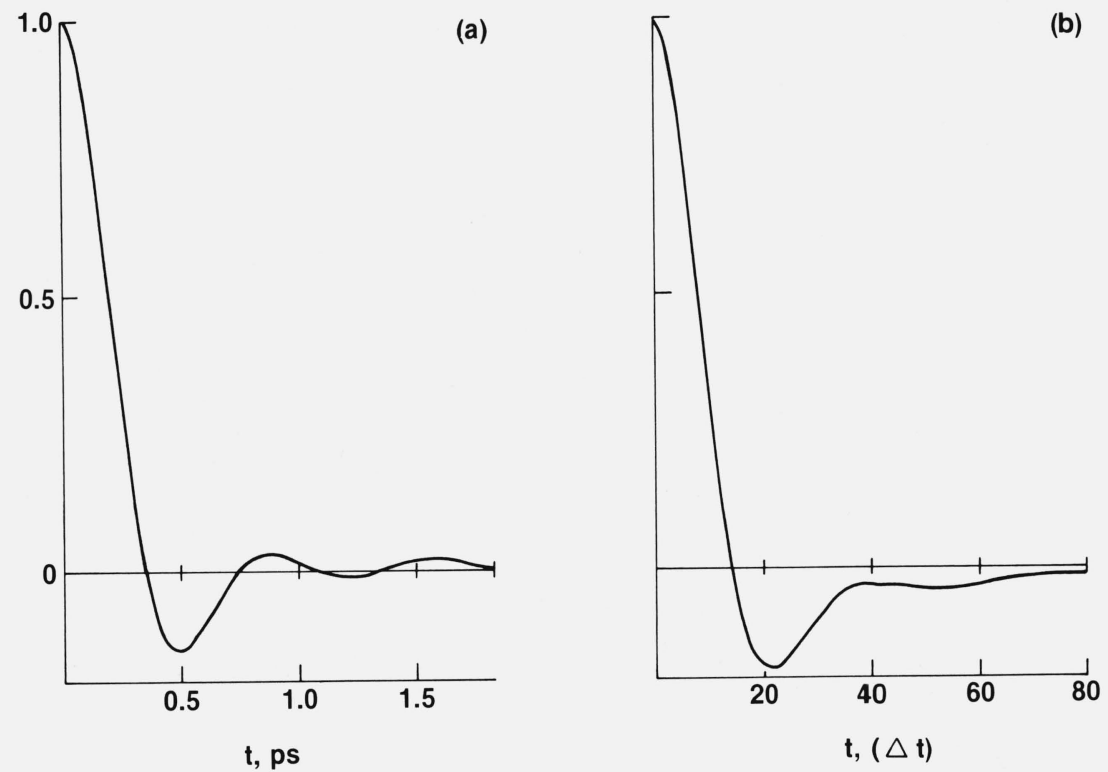

FIGURE 2. Velocity autocorrelation functions for (a)RB3 and (b) $L J 1$.

The rubidium functions contain damped oscillations about zero while the Lennard-Jones functions do not.

Our results for $C_{T}(Q, t) / C_{T}(Q, 0)$ are listed in tables $10-$ 14. We evaluated them by the same procedure used to construct $F(Q, t)$, as described above, except that the average includes two polarizations. The expected fluctuations about zero as $t \rightarrow \infty$ are on the order of 0.03 .

The trend of these functions with increasing $Q$ is shown in figure 3 for the states RB1 and RB3. For $Q$ 's much smaller than those available in present computer simulations, the longtime behavior of $C_{T}(Q, t)$ is expected to be

$$
C_{T}(Q, t) \propto \exp \left[-\eta Q^{2} t / m n\right]
$$

where $\eta$ is the shear viscosity of the liquid [1]. We have noted that the function displayed in figure $3 \mathrm{e}$ goes over to an

TAELE 10, TRANSUERSE CURRENT CORFELATION FUNCTIONS FOR RE1, THE TIME IS IN UNITS OF $\triangle T$.

WAVE VECTOR: D IN INUERSE ANGSTROM UNITS,

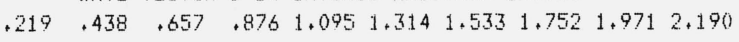$$
\text { TIME }
$$

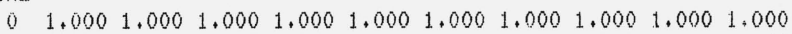

$\begin{array}{lllllllllll}.976 & .921 & .852 & .789 & .745 & .737 & .715 & .728 & .718 & .708\end{array}$

$\begin{array}{llllllllll}.911 & .718 & .495 & .319 & .230 & .215 & .186 & .227 & .246 & .210\end{array}$

$.820 \quad .457 \quad .105-.098-.161-.151-.137-.082-.061-.078$

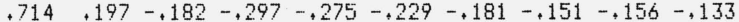

$.603-.020-.316-.291-.186-.132-.078-.082-.127-.087$

$.493-.179-.317-.178-.045-.008 \quad .010 \quad .003-.064-.015$

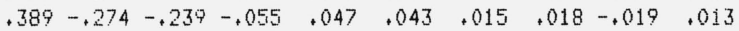

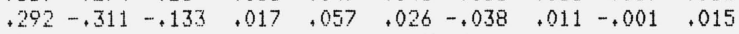

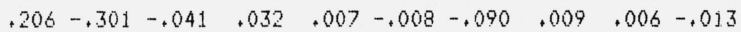

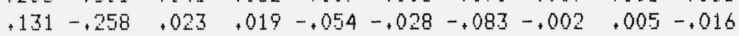

$.066-.196 \quad .054-.001-.085-.020-.057-.012 \quad .031 \quad .008$

$.011-.128 \quad .056-.010-.072-.001-.038-.020 \quad .042 \quad .011$

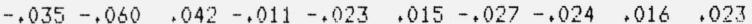

$\begin{array}{llllllllll}-.073 & -.003 & .024 & -.013 & .028 & .024 & -.028 & -.017 & -.000 & .034\end{array}$

$\begin{array}{llllllllll}-.103 & .040 & .010 & -.014 & .052 & .021 & -.009 & -.001 & -.010 & .042\end{array}$

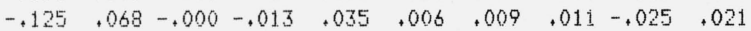

$\begin{array}{llllllllll}-.138 & .082 & -.006 & -.009 & -.000 & -.006 & .018 & .005 & -.025 & .008\end{array}$

$\begin{array}{llllllllll}-.144 & .086 & -.005 & -.007 & -.019 & -.007 & .029 & -.020 & -.010 & .001\end{array}$

$-.143 \quad .083-.001-.013-.007-.004 \quad .017-.030-.018 \quad .016$

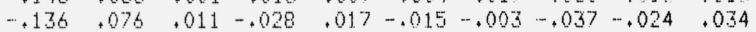

exponentially decaying form for $t>40 \Delta t$. An apparent viscosity of $0.28 \mathrm{cp}$ can be obtained by fitting this curve to an exponential for $t>40 \Delta t$. This value is consistent with reported values of the viscosity of liquid rubidium [16]. Before we could claim that this is the viscosity, it would be necessary to go to smaller $Q$ 's and show that $\eta$ is independent of $Q$. This would require a significantly larger system. While we cannot say with certainty, even in this best case, that we observed the small $Q$ regime, our results for intermediate $Q$ unambiguously show a change from a monotonically decaying $C_{T}(Q, t)$ at small $Q$ to damped oscillatory time dependence at larger $Q$. This transition is a gradual one and does not appear to depend strongly on the interatomic potential.

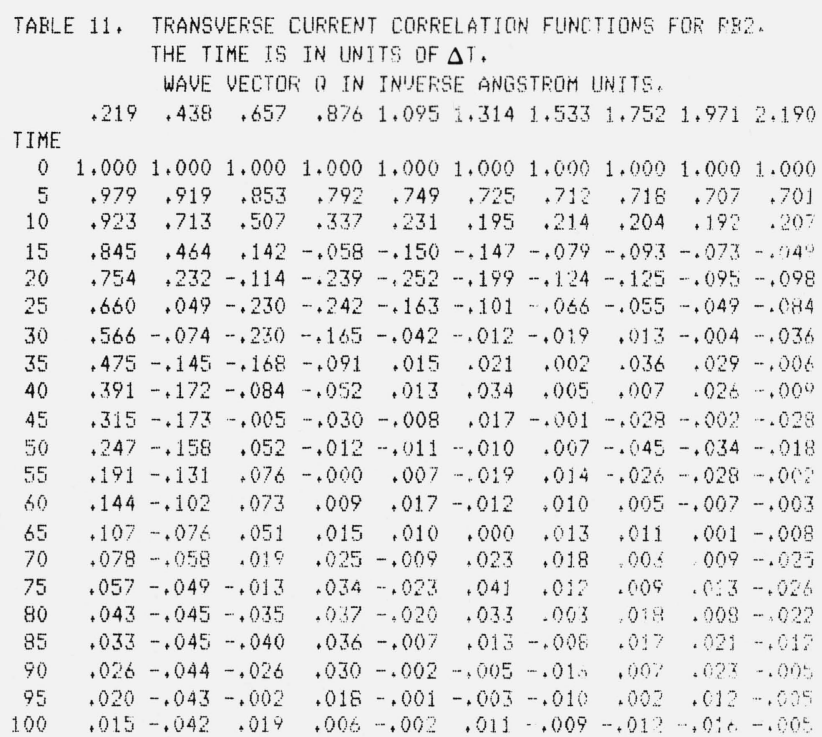


TAELE 12. TRIANSUEFSE CURERT CORRELATION FULCTIONS FOR REZ. THE TIME IS IN UNA IS UF $\triangle T$.

WAVE VECTOF Q I HE INUEFSE AHGSTROM UNTTS.

TIME

$.211+422+634.8461 .0561 .2673 .478 \quad 1.6901 .901 .2 .112$

() $1.0001 .000 \quad 1.000 \quad 1.000 \quad 1.000 \quad 1.000 \quad 1.000 \quad 1.000 \quad 1.000 \quad 1.000$

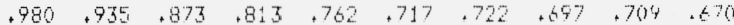

$\begin{array}{lllllllll}.930 \quad .773 & .579 & .419 & .292 & .213 & .253 & .219 & .233 & .172\end{array}$

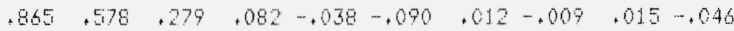

$.795 .395 .070-.082-.133 \cdots .137-.026-.072 \cdots .031-.072$

$.727 .243-.033-.103-.098-.050-.002-.047-.015-.030$

$.660 \quad .130-.066-.057-.034 \quad .022 \quad .032 \cdots .002-.013 \cdots+.009$

$\begin{array}{llllllll}.597 & .054-.064 \cdots .001 & -.001 \quad .037 \quad .052 & .017 & -.032 & \cdots .005\end{array}$

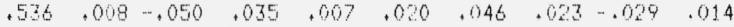

$.480-.018-.036 \quad .047 \quad .003-.012 .035 \quad .029+.024 \quad .029$

$.428-.028-.027 \quad .038 \quad .011-.014 \quad .014 \quad .019-.028 \quad .016$

$.382-.032-.019 \quad .023 \quad .020-.011-.005 \quad .011-.030-.004$

$.341-.033-.022 .008+.029-.020-.031-.004-.030-.025$

$.306-.031-.030 \quad .000 \quad .044-.017-.044-.012-.034-.028$

$.275-.027-.033-.005 \quad .048-.014-.023-.010-.023 \cdots .031$

$.248-.022-.029-.005 \quad .031-.013 \quad .001-.027 \quad .000-.029$

$.225-.017 \cdots .015 \quad .002-.008 \quad .004+.034-.017 \quad .005 \cdots .002$

$.203-.015-.003-.001-.031 \quad .024 \quad .043 \quad .005-.008-.003$

$.183-.017-.003-.015-.029 \quad .044 \quad .025-.001-.023 \quad .005$

$.166-.022-.014-.030-.023 \quad .034 \quad .019-.007-.030 \quad .008$

$.149-.029-.029-.029-.005-.000 \quad .004-.015-.008 \quad .027$
TABLE 13. TRANSUERSE CURRENT CORRELATIOR FUHCTIOKG FOE LIA. THE TIME IS IN UNITS OF $\Delta T$.

WAUE VECTOF IN REEIUCEII UNITS $(Q \sigma \sigma)$.

$.9801 .9612 .9413 .9224 .9025 .8836 .8637 .844 \quad 8.8249 .805$ TIME

$\begin{array}{llllllllll}0 & 1.000 & 1.000 & 1.000 & 1.000 & 1.000 & 1.000 & 1.000 & 1.0001 .0001 .000\end{array}$

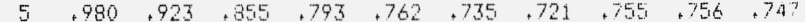

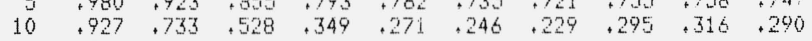

$15.857+503+200-.022-.079-.077-.077-.005 \quad .016+002$

$20 \quad .779 \quad .282 \cdots .033-.197 \cdots .187-.158 \cdots, 152 \cdots, 098 \cdots .090 \cdots, 096$

$25 \quad .699 \quad .098-.151-.213-.149-.103 \cdots .112-.080 \cdots .095 \cdots .072$

$30 \quad .618-.040-.180-.161-.066-.037-.036-.052 \cdots .087 \cdots .017$

$35 \quad .541-.136-.164-.118-.012-.006-.009-.040-.077 \quad .024$

$40 \quad .467-.194-.138-.085 \quad .003-.012-.011-.032-.051 \quad .036$

$45 \quad .399-.222-.109-.066-.004-.017-.007-.029-.013 \quad .026$

$50 \quad .336-.228-.085-.045-.028-.002 \quad .006-.017 .018+.010$

$55 \quad .280-.215-.059-.020-.044-.004 \quad .014 \quad .005 \quad .033-.015$

$60 \quad .230-.191-.034-.008-.031-.014 \quad .036 \quad .013-.007-.027$

$65 \quad .188-.157-.015-.010-.017-.035 \quad .050 \quad .022-.043-.037$

$70 \quad .151-.120-.003-.018-.017-.053 \quad .030 \quad .008 \cdots .049-.052$

$75 \quad .121-.082-.064-.026-.027-.037 \quad .012-.007-.036-.050$

$80 \quad .095-.050-.006-.020-.043-.008-.020-.022-.030-.035$

$85 \quad .072-.026-.008-.007-.034 \quad .004-.034-.028 \cdots .020-.026$

$90 \quad .053-.014-.001 \quad .010 \quad .003-.007-.013-.020 \cdots .007-.027$

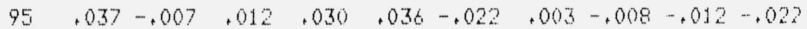

$\begin{array}{lllllll}100 \quad .025 & -.003 \quad .021 \quad .041 \quad .041 & -.032 \quad .017-.004 & -.004 & -.024\end{array}$

TABLE 14. TRANSUERSE CURFENT CORRELATION FUNCTIONS FOR LJ2.

THE TIME IS IN UNITS OF $\Delta$ T.

WAVE VECTOK IN FEIUICEI UNITS $(0 \boldsymbol{\sigma})$.

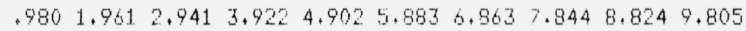

TIME

01.0001 .0001 .0001 .0001 .0001 .0001 .0001 .0001 .0001 .000

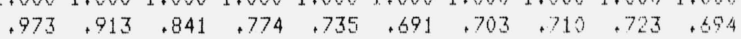

$\begin{array}{llllllllll}.906 & .708 & .497 & .322 & .222 & +188 & .197 & .234 & .262 & .211\end{array}$

$.819 .476 .174-.014-.089-.082-.078-.031-.025-.030$

$.724 .267-.039-.169-.145-.119-.134-.090-.121-.069$

$.627 .103-.148-.183-.091-.082-.112-.047-.116-.038$

$.533-.011-.189-.124-.045-.043-.065-.003-.078-.005$

$.443-.087-.180-.051-.035-.028-.045 \cdots .019-.058-.001$

$.360-.135-.142-.003-.040-.016-.039-.049-.046-.003$

$.282-.160-.082 .013-.048-.025-.018-.066-.033-.019$

$.211-.163-.029 \quad .029-.044-.025 \quad .011-.068-.019-.036$

$.146-.149 .001 \quad .032-.037-.016 \quad .012-.029 \quad .010-.025$

$.089-.119 \quad .018 \quad .023-.057 \quad .009-.006-.004 \quad .026-.027$

$.038-.080 \quad .022 \quad .017-.061 \quad .025-.029-.025 \quad .028-.013$

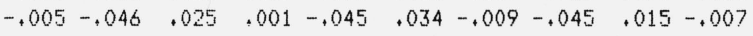

$-.042-.017 \quad .012-.014-.027 \quad .038 \quad .019-.057-.006 \quad .008$

$80-.072-.003-.004-.014 \quad .005 \quad .027 \quad .016-.050-.019 \quad .027$

$85-.096 \quad .007-.015-.023 \quad .033 \quad .005 \quad .013-.026-.030 \quad .006$

$90 \quad-.114 \quad .006-.017-.035 \quad .039-.021 \quad .001 \quad .002-.032-.020$

$95-.126 \quad .008-.008-.038 \quad .034-.030-.006 \quad .016-.032-.026$

$100 \quad-.133 \quad .007-.000-.037 \quad .017-.017-.004 \quad .020-.024-.017$

\section{Concluding Remarks}

The dynamical properties of the two fluids considered here are quite different. The most obvious difference is the striking disparity in the spectra of density fluctuations. However, the results which we have obtained for the other functions, especially the velocity autocorrelation function, confirm previous suggestions that the difference is more pervasive. In general, liquid rubidium appears to be more resilient and elastic than the Lennard-Jones fluid. While it is plausible that this behavior would result from the softer core of the potential in rubidium, formulating a theory which quantifies this intuition remains as an outstanding problem in the theory of liquids. 


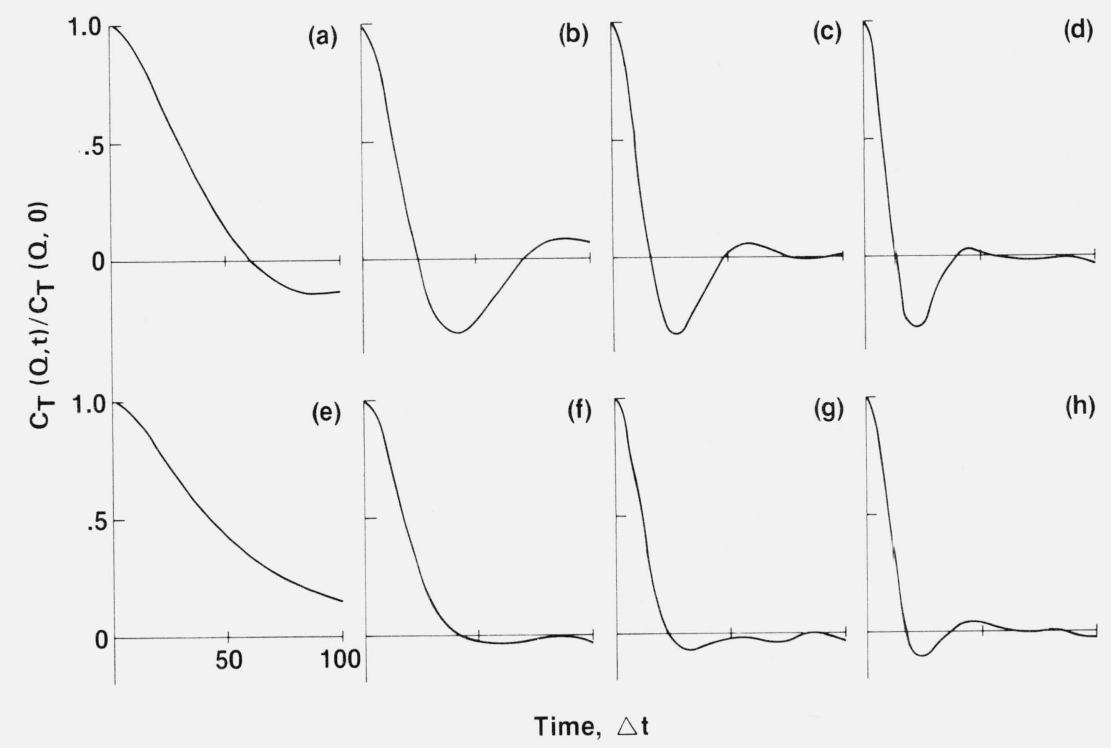

FIgURE 3. Normalized transverse current correlation functions for $R B 1(a-d)$ and $R B 3(e-h)$.

The $Q$ values are (a) $.219 \AA^{-1}$; (b) $.438 \AA^{-1}$; (c) $.657 \AA^{-1}$; (d) $.876 \AA^{-1}$, (e) $.211 \AA^{-1}$; (f) $.422 \AA^{-1}$; (g) $.634 \AA^{-1}$; (h) $.845 \AA^{-1}$. These functions show the effect of increasing $Q$ on the transverse current correlations. Similar results obtain for the Lennard-Jones fluids as is indicated in tables 13-14.

\section{References}

[1] Mountain, R. D. Dynamics of Solids and Liquids by Neutron Scattering, eds. S. W. Lovesey and T. Springer (Springer-Verlag, New York, 1977) Ch. 6.

[2] Copley, J. R. D., and Rowe, J. M. Phys. Rev. Letters 32, 49 (1974), Phys. Rev. A9, 1656 (1974).

[3] Rahman, A. Phys. Rev. Letters 32, 52 (A74), Phys. Rev. A9, 1667 (1974).

[4] Bell, H., Moeller-Wenghoffer, H., Kollmar, A., Stockmeyer, R., Springer, T., and Stiller, H. Phys. Rev. A11, 316 (1975).

[5] Levesque, D., Verlet, L., and Kurkijarvi, J. Phys. Rev. A7, 1690 (1973).

[6] Mountain, R. D., and Haan, S. W. Phys. Rev. A.

[7] Haan, S. W. Phys. Rev. A.
[8] Price, D. L. Phys. Rev. A4, 353 (1971); Price, D. L., Singwi, K. S., and Tosi, M. P. Phys. Rev. B 2, 2983 (1976).

[9] Mountain, R. D., J. Phys. F: Metal Physics 8, 1637 (1978).

[10] Tsai, D. H. Accurate Characterization of the High Pressure Environment, ed. E. C. Lloyd, Nat. Bur. Stand. (U.S.) Spec. Publ. 326 p. 105, (March 1971). See Also Tsai, D. H., Bullough, R., and Perrin, R. C. J. Phys. C: Solid State Phys. 3, 2022 (1970).

[11] Streett, W. B., Raveché, H. J., and Mountain, R. D. J. Chem. Phys. 61, 1960 (1974). The rubidium energies were taken from our unpublished calculations.

[12] Rahman, A., Phys. Rev. 136, A 405 (1964).

[13] Schiff, D., Phys. Rev. 186, 151 (1969).

[14] Gaskell, T., and Miller, S., Phys. Letters 66A, 307 (1978).

[15] Rahman, A. Phys. Rev. Letters 19, 240 (1967) and in Neutron Inelastic Scattering, Vol. 1 (IAEA, Vienna, 1968) p. 561.

[16] Andrade, E. N. daC., and Dobbs, E. R. Proc. Roy. Soc. London 211 , 12 (1952). 